\title{
Simple Energy Corrections for Precise Atomization Energies of $\mathrm{CHON}$ Molecules \\ Pablo F. Salazar and Jorge M. Seminario \\ Department of Chemical Engineering and \\ Department of Electrical and Computer Engineering \\ Texas A\&M University \\ College Station, Texas \\ USA
}

Table A1. Atomization energies for several levels of theory and their experimental values

\begin{tabular}{|c|c|c|c|c|c|c|c|c|}
\hline Molecule & Name & $\begin{array}{l}\text { G3x } \\
\text { (Ha) }\end{array}$ & $\begin{array}{c}\text { B3PW91/ } \\
\text { 6-31G** } \\
\text { (Ha) }\end{array}$ & $\begin{array}{c}\text { B3PW91/ } \\
\text { cc-pVTZ } \\
\text { (Ha) }\end{array}$ & $\begin{array}{c}\mathrm{HF} / \\
6-31 \mathrm{G}^{*}(\mathrm{Ha})\end{array}$ & $\begin{array}{c}\mathrm{HF} / \\
3-21 \mathrm{G}(\mathrm{Ha})\end{array}$ & $\begin{array}{c}\text { MP2(full)/ } \\
\text { 6-31G** } \\
\text { (Ha) }\end{array}$ & $\begin{array}{c}\text { Exp. } \\
\text { Atomization } \\
\text { energy } \\
(\mathrm{kcal} / \mathrm{mol})^{a}\end{array}$ \\
\hline \multicolumn{9}{|l|}{ TRAINING SET } \\
\hline $\mathrm{H}_{2}$ & Hydrogen gas & 103.7 & 102.3 & 101.0 & 75.2 & 75.3 & 86.2 & 103.5 \\
\hline $\mathrm{CH}\left({ }^{2} \mathrm{P}\right)$ & Methylidyne & 81.4 & 78.6 & 79.4 & 49.5 & 42.7 & 63.4 & 79.9 \\
\hline $\mathrm{CH}_{2}\left({ }^{3} \mathrm{~B}_{1}\right)$ & Methylene & 180.9 & 177.5 & 183.2 & 141.7 & 136.5 & 160.9 & 179.6 \\
\hline $\mathrm{CH}_{2}\left({ }^{1} \mathrm{~A}_{1}\right)$ & Methylene & 171.6 & 165.7 & 166.9 & 111.1 & 100.9 & 140.4 & 170.6 \\
\hline $\mathrm{CH}_{3}\left({ }^{2} \mathrm{~A}_{2}\right)$ & Methyl radical & 289.8 & 292.1 & 290.7 & 221.2 & 214.5 & 258.1 & 289.2 \\
\hline $\mathrm{CH}_{4}$ & Methane & 392.5 & 394.8 & 391.5 & 297.2 & 290.5 & 352.2 & 392.5 \\
\hline $\mathrm{NH}\left({ }^{3} \mathrm{~S}_{\mathrm{g}}\right)$ & Imidogen & 80.1 & 79.6 & 80.5 & 42.5 & 31.7 & 60.3 & 79.0 \\
\hline $\mathrm{NH}_{2}\left({ }^{2} \mathrm{~B}_{1}\right)$ & Amino radical & 170.8 & 169.2 & 171.1 & 97.4 & 80.3 & 136.9 & 170.0 \\
\hline NH3 & Ammonia & 276.0 & 271.3 & 273.6 & 167.7 & 152.0 & 230.9 & 276.7 \\
\hline $\mathrm{OH}$ & Hydroxyl radical & 102.2 & 98.8 & 100.2 & 57.1 & 45.3 & 84.4 & 101.3 \\
\hline $\mathrm{OH}_{2}$ & Water & 219.0 & 210.4 & 213.4 & 130.1 & 111.8 & 187.8 & 219.3 \\
\hline $\mathrm{HCCH}$ & Acetylene & 388.6 & 384.0 & 386.8 & 270.0 & 258.1 & 364.5 & 388.9 \\
\hline $\mathrm{H}_{2} \mathrm{CCH}_{2}$ & Ethylene & 532.0 & 533.5 & 531.7 & 390.5 & 375.9 & 487.1 & 531.9 \\
\hline $\mathrm{H}_{3} \mathrm{CCH}_{3}$ & Ethane & 666.1 & 671.5 & 665.6 & 500.7 & 486.0 & 605.1 & 666.3 \\
\hline $\mathrm{CN}(2)$ & Cyano radical & 178.5 & 172.8 & 175.4 & 84.1 & 59.1 & 150.3 & 176.6 \\
\hline $\mathrm{HCN}$ & Hydrogen cyanide & 303.4 & 298.9 & 301.8 & 183.7 & 158.8 & 286.6 & 301.8 \\
\hline $\mathrm{CO}$ & Carbon monoxide & 257.0 & 249.9 & 252.2 & 167.9 & 133.8 & 254.0 & 256.2 \\
\hline $\mathrm{HCO}\left({ }^{2} \mathrm{~A}^{\prime}\right)$ & Formyl radical & 271.3 & 271.2 & 272.3 & 169.6 & 137.3 & 266.1 & 270.3 \\
\hline $\mathrm{H}_{2} \mathrm{CO}$ & Formaldehyde & 358.2 & 356.7 & 356.3 & 235.9 & 204.4 & 351.7 & 357.2 \\
\hline $\mathrm{H}_{3} \mathrm{COH}$ & Methyl alcohol & 481.0 & 479.0 & 477.7 & 327.8 & 303.7 & 432.5 & 480.8 \\
\hline $\mathrm{N}_{2}$ & Nitrogen gas & 224.8 & 215.6 & 221.5 & 104.7 & 52.8 & 211.9 & 225.1 \\
\hline $\mathrm{H}_{2} \mathrm{NNH}_{2}$ & Hydrazine & 403.9 & 400.4 & 403.4 & 218.1 & 187.1 & 337.5 & 405.4 \\
\hline $\mathrm{NO}(2)$ & Nitric oxide & 150.0 & 148.1 & 150.7 & 46.1 & -0.1 & 134.4 & 150.1 \\
\hline $\mathrm{O}_{2}\left({ }^{3} \mathrm{~S}_{\mathrm{gg}}\right)$ & Oxygen gas & 118.0 & 123.0 & 122.7 & 28.5 & -13.8 & 117.4 & 118.0 \\
\hline $\mathrm{HOOH}$ & Hydrogen peroxide & 251.5 & 246.2 & 247.7 & 107.4 & 87.3 & 218.5 & 252.3 \\
\hline $\mathrm{CO}_{2}$ & Carbon dioxide & 383.6 & 380.8 & 383.8 & 233.9 & 176.3 & 380.5 & 381.9 \\
\hline $\mathrm{CH}_{2}=\mathrm{C}=\mathrm{CH}_{2}$ & Allene & 670.7 & 671.1 & 670.9 & 482.4 & 462.2 & 626.3 & 669.1 \\
\hline $\mathrm{CH}_{3} \mathrm{CCH}$ & Propyne & 669.6 & 674.0 & 673.1 & 480.8 & 459.4 & 621.1 & 670.6 \\
\hline $\mathrm{C}_{3} \mathrm{H}_{4}$ & Cyclopropene & 646.0 & 652.6 & 650.3 & 456.2 & 422.5 & 602.3 & 648.1 \\
\hline $\mathrm{CH}_{3} \mathrm{CH}=\mathrm{CH}_{2}$ & Propylene & 811.2 & 816.0 & 811.5 & 598.7 & 575.9 & 746.2 & 811.3 \\
\hline $\mathrm{C}_{3} \mathrm{H}_{6}$ & Cyclopropane & 802.1 & 810.8 & 805.3 & 589.6 & 560.7 & 740.5 & 802.9 \\
\hline $\mathrm{C}_{3} \mathrm{H}_{8}$ & Propane & 942.6 & 950.0 & 941.5 & 705.7 & 683.7 & 861.1 & 942.6 \\
\hline $\mathrm{CH}_{2} \mathrm{CHCHCH}_{2}$ & Butadiene & 959.5 & 964.5 & 961.0 & 699.8 & 665.6 & 891.0 & 959.9 \\
\hline $\mathrm{C}_{4} \mathrm{H}_{6}$ & 2-butyne & 951.8 & 957.1 & 953.8 & 693.9 & 643.3 & 887.5 & 952.1 \\
\hline
\end{tabular}




\begin{tabular}{|c|c|c|c|c|c|c|c|c|}
\hline Molecule & Name & $\begin{array}{l}\text { G3x } \\
\text { (Ha) }\end{array}$ & $\begin{array}{c}\text { B3PW91/ } \\
\text { 6-31G** } \\
(\mathrm{Ha})\end{array}$ & $\begin{array}{c}\text { B3PW91/ } \\
\text { cc-pVTZ } \\
\text { (Нa) }\end{array}$ & $\begin{array}{c}\mathrm{HF} / \\
6-31 \mathrm{G}^{*}(\mathrm{Ha})\end{array}$ & $\begin{array}{c}\mathrm{HF} / \\
3-21 \mathrm{G}(\mathrm{Ha})\end{array}$ & $\begin{array}{c}\text { MP2(full)/ } \\
\text { 6-31G* } \\
\text { (Ha) }\end{array}$ & $\begin{array}{c}\text { Exp. } \\
\text { Atomization } \\
\text { energy } \\
(\mathrm{kcal} / \mathrm{mol})^{\mathrm{a}}\end{array}$ \\
\hline $\mathrm{C}_{4} \mathrm{H}_{6}$ & Methylene cyclopropane & 939.6 & 949.6 & 944.9 & 679.4 & 643.3 & 874.3 & 938.0 \\
\hline $\mathrm{C}_{4} \mathrm{H}_{6}$ & Bicyclobutane & 931.3 & 943.1 & 937.1 & 668.8 & 622.5 & 869.3 & 933.7 \\
\hline $\mathrm{C}_{4} \mathrm{H}_{6}$ & Cyclobutene & 946.3 & 955.8 & 950.0 & 686.2 & 650.2 & 881.9 & 948.2 \\
\hline $\mathrm{C}_{4} \mathrm{H}_{8}$ & Cycloctane & 1080.1 & 1091.3 & 1081.8 & 796.4 & 764.9 & 998.4 & 1080.3 \\
\hline $\mathrm{C}_{4} \mathrm{H}_{8}$ & Isobutene & 1091.7 & 1098.5 & 1091.3 & 806.8 & 776.4 & 1006.4 & 1091.8 \\
\hline $\mathrm{C}_{4} \mathrm{H}_{10}$ & Trans butane & 1219.4 & 1228.6 & 1217.6 & 910.8 & 881.4 & 1117.3 & 1219.3 \\
\hline $\mathrm{C}_{5} \mathrm{H}_{8}$ & Spiropentane & 1212.8 & 1227.8 & 1219.3 & 880.9 & 830.0 & 1131.1 & 1213.0 \\
\hline $\mathrm{CH}_{3} \mathrm{CN}$ & Methyl cyanide & 588.7 & 587.7 & 587.9 & 399.6 & 367.9 & 551.0 & 587.7 \\
\hline $\mathrm{CH}_{3} \mathrm{ONO}$ & Methyl nitrite & 569.3 & 571.0 & 569.1 & 305.0 & 233.5 & 523.8 & 568.2 \\
\hline $\mathrm{HCOOH}$ & Formic acid & 480.4 & 478.4 & 478.6 & 301.2 & 253.1 & 452.2 & 479.9 \\
\hline $\mathrm{NCCN}$ & Cyanogen & 493.7 & 487.1 & 492.3 & 276.4 & 232.6 & 481.4 & 492.1 \\
\hline$\left(\mathrm{CH}_{3}\right)_{2} \mathrm{NH}$ & Dimethylamine & 812.4 & 816.2 & 811.3 & 567.6 & 536.6 & 729.6 & 813.0 \\
\hline $\mathrm{CH}_{2} \mathrm{CO}$ & Ketene & 513.9 & 515.7 & 517.2 & 344.1 & 309.7 & 487.3 & 512.9 \\
\hline $\mathrm{C}_{2} \mathrm{H}_{4} \mathrm{O}$ & Oxirane & 615.3 & 619.1 & 616.0 & 418.2 & 375.7 & 570.3 & 615.1 \\
\hline $\mathrm{CH}_{3} \mathrm{CHO}$ & Acetaldehyde & 643.0 & 645.3 & 642.4 & 450.2 & 410.9 & 598.7 & 642.6 \\
\hline $\mathrm{HCOCOH}$ & Glyoxal & 612.2 & 611.1 & 610.2 & 392.4 & 329.3 & 585.3 & 610.5 \\
\hline $\mathrm{CH}_{2}=\mathrm{CHCN}$ & Acrylonitrile & 731.8 & 731.1 & 732.0 & 495.9 & 456.6 & 691.1 & 732.5 \\
\hline $\mathrm{CH}_{3} \mathrm{COOH}$ & Acetic acid & 764.9 & 765.4 & 763.0 & 515.3 & 460.6 & 715.9 & 764.7 \\
\hline$\left(\mathrm{CH}_{3}\right)_{2} \mathrm{CHOH}$ & Isopropanol & 1041.7 & 1043.5 & 1036.6 & 744.9 & 706.9 & 952.6 & 1041.3 \\
\hline$\left(\mathrm{CH}_{3}\right)_{3} \mathrm{~N}$ & Trimethylamine & 1086.4 & 1091.9 & 1083.8 & 770.4 & 732.2 & 984.8 & 1086.0 \\
\hline $\mathrm{C}_{4} \mathrm{H}_{4} \mathrm{O}$ & Furan & 950.5 & 958.0 & 955.1 & 658.4 & 609.7 & 904.4 & 950.6 \\
\hline $\mathrm{C}_{4} \mathrm{H}_{5} \mathrm{~N}$ & Pyrrole & 1019.9 & 1028.1 & 1026.8 & 702.2 & 657.2 & 956.5 & 1020.7 \\
\hline $\mathrm{C}_{5} \mathrm{H}_{5} \mathrm{~N}$ & Pyridine & 1183.1 & 1194.3 & 1190.7 & 828.0 & 768.0 & 1121.9 & 1182.9 \\
\hline $\mathrm{CCH}\left({ }^{2} \mathrm{~A}^{\prime}\right)$ & Ethynyl radical & 257.9 & 253.9 & 256.3 & 172.6 & 162.4 & 228.8 & 257.5 \\
\hline $\mathrm{C}_{2} \mathrm{H}_{3}\left({ }^{2} \mathrm{~A}^{\prime}\right)$ & Vinyl & 423.5 & 426.4 & 426.1 & 310.7 & 297.0 & 380.7 & 422.3 \\
\hline $\mathrm{CH}_{3} \mathrm{CO}\left({ }^{2} \mathrm{~A}^{\prime}\right)$ & Acetyl radical & 555.1 & 558.9 & 557.6 & 383.5 & 343.8 & 519.9 & 554.7 \\
\hline $\mathrm{H}_{2} \mathrm{COH}\left({ }^{2} \mathrm{~A}\right)$ & Hydroxymethyl radical & 386.2 & 386.6 & 387.0 & 256.7 & 233.1 & 346.2 & 386.3 \\
\hline $\mathrm{C}_{2} \mathrm{H}_{5}\left({ }^{2} \mathrm{~A}^{\prime}\right)$ & Ethyl radical & 566.8 & 573.7 & 569.7 & 427.3 & 412.5 & 513.9 & 566.7 \\
\hline$\left(\mathrm{CH}_{3}\right)_{2} \mathrm{CH}\left({ }^{2} \mathrm{~A}^{\prime}\right)$ & Isopropyl radical & 845.8 & 856.2 & 849.6 & 634.6 & 611.8 & 772.3 & 845.8 \\
\hline$\left(\mathrm{CH}_{3}\right)_{3} \mathrm{C}\left({ }^{2} \mathrm{~A}_{1}\right)$ & t-butyl radical & 1126.0 & 1138.8 & 1129.3 & 842.3 & 811.8 & 1032.1 & 1126.8 \\
\hline $\mathrm{NO}_{2}(2)$ & Nitrogen dioxide & 222.5 & 225.5 & 228.5 & 42.9 & -35.6 & 210.3 & 221.9 \\
\hline $\mathrm{C}_{4} \mathrm{H}_{6}$ & Methyl allene & 947.8 & 955.2 & 951.4 & 687.9 & 658.5 & 879.4 & 947.6 \\
\hline $\mathrm{C}_{5} \mathrm{H}_{8}$ & Isoprene & 1239.6 & 1246.0 & 1239.8 & 906.8 & 866.4 & 1150.6 & 1239.9 \\
\hline $\mathrm{C}_{5} \mathrm{H}_{10}$ & Cyclopentane & 1376.2 & 1388.1 & 1376.0 & 1020.5 & 985.4 & 1274.2 & 1377.0 \\
\hline $\mathrm{C}_{5} \mathrm{H}_{12}$ & n-pentane & 1496.1 & 1507.2 & 1493.5 & 1115.9 & 1079.1 & 1373.4 & 1496.2 \\
\hline $\mathrm{C}_{5} \mathrm{H}_{12}$ & Neo pentane & 1501.0 & 1508.5 & 1494.7 & 1117.0 & 1083.0 & 1379.0 & 1501.1 \\
\hline $\mathrm{C}_{6} \mathrm{H}_{8}$ & 1,3 cyclohexadiene & 1401.1 & 1411.4 & 1403.7 & 1022.4 & 975.9 & 1313.5 & 1401.8 \\
\hline $\mathrm{C}_{6} \mathrm{H}_{8}$ & 1,4 cyclohexadiene & 1401.1 & 1411.3 & 1404.0 & 1023.5 & 976.2 & 1312.3 & 1402.2 \\
\hline $\mathrm{C}_{6} \mathrm{H}_{12}$ & Cyclohexane & 1659.1 & 1671.9 & 1656.6 & 1230.9 & 1188.1 & 1536.5 & 1659.5 \\
\hline $\mathrm{C}_{6} \mathrm{H}_{14}$ & n-hexane & 1773.0 & 1785.8 & 1769.6 & 1321.0 & 1276.8 & 1629.7 & 1772.8 \\
\hline $\mathrm{C}_{6} \mathrm{H}_{14}$ & 3-methyl pentane & 1773.8 & 1784.8 & 1768.4 & 1318.9 & 1276.1 & 1630.2 & 1774.0 \\
\hline $\mathrm{C}_{6} \mathrm{H}_{5} \mathrm{CH}_{3}$ & Toluene & 1585.3 & 1599.8 & 1591.8 & 1161.7 & 1107.7 & 1501.2 & 1585.6 \\
\hline $\mathrm{C}_{7} \mathrm{H}_{16}$ & n-heptane & 2049.8 & 2064.4 & 2045.5 & 1526.1 & 1474.5 & 1885.9 & 2049.6 \\
\hline $\mathrm{C}_{8} \mathrm{H}_{8}$ & Cyclooctatetraene & 1696.0 & 1705.9 & 1699.8 & 1222.4 & 1157.9 & 1602.8 & 1696.9 \\
\hline $\mathrm{C}_{8} \mathrm{H}_{18}$ & n-octane & 2326.7 & 2343.1 & 2321.6 & 1731.2 & 1672.3 & 2142.2 & 2326.5 \\
\hline $\mathrm{C}_{10} \mathrm{H}_{8}$ & Naphthalene & 2072.1 & 2092.4 & 2083.9 & 1507.5 & 1428.1 & 1987.7 & 2071.2 \\
\hline $\mathrm{C}_{10} \mathrm{H}_{8}$ & Azulene & 2036.7 & 2058.9 & 2050.6 & 1464.3 & 1381.6 & 1858.5 & 2038.2 \\
\hline
\end{tabular}




\begin{tabular}{|c|c|c|c|c|c|c|c|c|}
\hline Molecule & Name & $\begin{array}{l}\text { G3x } \\
(\mathrm{Ha})\end{array}$ & $\begin{array}{c}\text { B3PW91/ } \\
6-31 \mathrm{G}^{* *} \\
\text { (Ha) }\end{array}$ & $\begin{array}{l}\text { B3PW91/ } \\
\text { cc-pVTZ } \\
\text { (Ha) }\end{array}$ & $\begin{array}{c}\mathrm{HF} / \\
6-31 \mathrm{G}^{*}(\mathrm{Ha})\end{array}$ & $\begin{array}{c}\mathrm{HF} / \\
3-21 \mathrm{G}(\mathrm{Ha})\end{array}$ & $\begin{array}{l}\text { MP2(full)/ } \\
\text { 6-31G* } \\
\text { (Ha) }\end{array}$ & $\begin{array}{c}\text { Exp. } \\
\text { Atomization } \\
\text { energy } \\
(\mathrm{kcal} / \mathrm{mol})^{\mathrm{a}} \\
\end{array}$ \\
\hline $\mathrm{CH}_{3} \mathrm{COOCH}_{3}$ & Acetic acid methyl ester & 1032.7 & 1036.0 & 1030.0 & 715.3 & 654.8 & 965.1 & 1031.7 \\
\hline$\left(\mathrm{CH}_{3}\right)_{3} \mathrm{COH}$ & t-butanol & 1323.4 & 1325.2 & 1315.4 & 952.7 & 909.2 & 1214.1 & 1322.6 \\
\hline $\mathrm{C}_{6} \mathrm{H}_{5} \mathrm{NH}_{2}$ & Aniline & 1467.3 & 1478.8 & 1475.2 & 1033.5 & 974.2 & 1380.2 & 1467.6 \\
\hline $\mathrm{C}_{6} \mathrm{H}_{5} \mathrm{OH}$ & Phenol & 1406.3 & 1416.6 & 1412.3 & 996.0 & 935.0 & 1335.5 & 1407.1 \\
\hline $\mathrm{C}_{4} \mathrm{H}_{6} \mathrm{O}$ & Divinyl ether & 1048.0 & 1052.4 & 1049.3 & 732.8 & 687.6 & 975.3 & 1048.2 \\
\hline $\mathrm{C}_{4} \mathrm{H}_{8} \mathrm{O}$ & Tetrahydrofuran & 1189.4 & 1197.0 & 1187.8 & 851.7 & 807.1 & 1103.4 & 1189.5 \\
\hline $\mathrm{C}_{5} \mathrm{H}_{8} \mathrm{O}$ & Cyclopentanone & 1362.4 & 1372.5 & 1362.8 & 980.0 & 921.9 & 1277.1 & 1361.5 \\
\hline $\mathrm{C}_{6} \mathrm{H}_{4} \mathrm{O}_{2}$ & Benzoquinone & 1370.4 & 1378.0 & 1373.6 & 939.5 & 846.6 & 1317.9 & 1370.7 \\
\hline $\mathrm{C}_{4} \mathrm{H}_{4} \mathrm{~N}_{2}$ & Pyrimidine & 1063.3 & 1072.5 & 1070.7 & 704.7 & 629.3 & 1004.3 & 1060.8 \\
\hline $\mathrm{N}=\mathrm{C}-\mathrm{CH}_{2}-\mathrm{CH}_{2}-\mathrm{C}=\mathrm{N}$ & Butanedinitrile & 1060.3 & 1057.1 & 1057.9 & 704.9 & 642.1 & 1005.1 & 1058.7 \\
\hline $\mathrm{C}_{4} \mathrm{H}_{4} \mathrm{~N}_{2}$ & 1,4-dipyridine & 1058.7 & 1068.4 & 1066.6 & 698.3 & 624.0 & 1000.8 & 1060.7 \\
\hline $\mathrm{CH}_{3}-\mathrm{C}(=\mathrm{O})-\mathrm{CCH}$ & & 926.1 & 926.8 & 925.8 & 638.8 & 588.2 & 879.0 & 927.9 \\
\hline $\mathrm{CH}_{3}-\mathrm{CH}=\mathrm{CH}-\mathrm{CHO}$ & Crotonaldehyde & 1070.0 & 1076.0 & 1071.2 & 759.6 & 704.8 & 1002.3 & 1068.8 \\
\hline $\mathrm{CH}_{3}-\mathrm{C}(=\mathrm{O})-\mathrm{O}-\mathrm{C}(=\mathrm{O})-\mathrm{CH}_{3}$ & Acetic anhydride & 1301.8 & 1305.7 & 1299.2 & 886.3 & 797.2 & 1232.6 & 1299.0 \\
\hline$\left(\mathrm{CH}_{3}\right)_{2} \mathrm{CH}-\mathrm{CN}$ & Propanenitrile, 2-methyl & 1143.1 & 1145.1 & 1139.9 & 810.3 & 764.9 & 1064.6 & 1143.5 \\
\hline $\mathrm{CH}_{3}-\mathrm{CO}-\mathrm{CH}_{2}-\mathrm{CH}_{3}$ & Methyl ethyl ketone & 1204.1 & 1211.0 & 1202.5 & 944.9 & 816.4 & 1119.0 & 1203.7 \\
\hline$\left(\mathrm{CH}_{3}\right)_{2} \mathrm{CH}-\mathrm{CHO}$ & Propanal,2-methyl & 1197.5 & 1202.7 & 1194.5 & 860.6 & 808.5 & 1112.2 & 1198.0 \\
\hline $\mathrm{C}_{4} \mathrm{H}_{8} \mathrm{O}_{2}$ & 1,4-Dioxane & 1280.6 & 1285.2 & 1275.5 & 888.2 & 826.6 & 1190.2 & 1279.1 \\
\hline $\mathrm{C}_{4} \mathrm{H}_{8} \mathrm{NH}$ & Tetrahydropyrrole & 1250.0 & 1258.1 & 1249.8 & 886.6 & 841.6 & 1146.7 & 1250.5 \\
\hline $\mathrm{CH}_{3}-\mathrm{CH}_{2}-\mathrm{CH}\left(\mathrm{CH}_{3}\right)-\mathrm{NO}_{2}$ & Nitro-s-butane & 1408.5 & 1414.5 & 1405.8 & 921.0 & 816.4 & 1303.2 & 1406.6 \\
\hline $\mathrm{CH}_{3}-\mathrm{CH}_{2}-\mathrm{O}-\mathrm{CH}_{2}-\mathrm{CH}_{3}$ & Diethyl ether & 1309.0 & 1315.5 & 1305.0 & 946.5 & 900.6 & 1202.5 & 1308.2 \\
\hline $\mathrm{CH}_{3}-\mathrm{CH}\left(\mathrm{OCH}_{3}\right)_{2}$ & dimethyl acetal & 1401.7 & 1404.5 & 1393.4 & 978.1 & 917.2 & 1285.1 & 1399.6 \\
\hline $\begin{array}{l}\left(\mathrm{CH}_{3}\right)_{3} \mathrm{C}-\mathrm{NH}_{2} \\
\text { cyc-CH=CH-N }\left(\mathrm{CH}_{3}\right)-\end{array}$ & t-butylamine & 1381.2 & 1384.3 & 1374.7 & 987.0 & 943.9 & 1256.2 & 1381.0 \\
\hline $\mathrm{CH}=\mathrm{CH}-$ & $\mathrm{N}$-methyl pyrrole & 1293.6 & 1303.8 & 1299.5 & 905.4 & 852.2 & 1211.6 & 1294.0 \\
\hline $\mathrm{C}_{5} \mathrm{H}_{10} \mathrm{O}$ & Tetrahydropyran & 1470.5 & 1479.3 & 1466.9 & 1060.3 & 1008.6 & 1364.2 & 1470.2 \\
\hline $\mathrm{CH}_{3}-\mathrm{CH}_{2}-\mathrm{CO}-\mathrm{CH}_{2}-\mathrm{CH}_{3}$ & Diethyl ketone & 1481.2 & 1490.1 & 1478.7 & 1074.0 & 1015.6 & 1375.6 & 1480.0 \\
\hline $\mathrm{C}_{5} \mathrm{H}_{10} \mathrm{O}_{2}$ & Isopropyl acetate & 1593.4 & 1599.7 & 1588.1 & 1131.6 & 1057.6 & 1485.4 & 1591.9 \\
\hline cyc- $\mathrm{C}_{5} \mathrm{H}_{10} \mathrm{NH}$ & Piperidine & 1531.7 & 1541.4 & 1529.4 & 1096.5 & 1044.1 & 1408.6 & 1532.4 \\
\hline$\left(\mathrm{CH}_{3}\right)_{2} \mathrm{CH}-\mathrm{O}-\mathrm{CH}\left(\mathrm{CH}_{3}\right)_{2}$ & Di-isopropyl ether & 1869.2 & 1877.2 & 1860.7 & 1360.4 & 1302.2 & 1722.2 & 1867.8 \\
\hline $\mathrm{C}_{6} \mathrm{H}_{5}\left({ }^{2} \mathrm{~A}_{1}\right)$ & Phenyl radical & 1192.4 & 1209.0 & 1205.0 & 876.7 & 830.6 & 1113.7 & 1193.6 \\
\hline$\left(\mathrm{CH}_{3}\right)_{3} \mathrm{C}-\mathrm{O}-\mathrm{CH}_{3}$ & t-butyl methyl ether & 1588.9 & 1593.8 & 1580.0 & 1149.9 & 1100.7 & 1462.0 & 1587.6 \\
\hline $\mathrm{C}_{6} \mathrm{H}_{6}$ & Benzene & 1305.2 & 1317.8 & 1312.7 & 954.4 & 908.0 & 1306.0 & 1305.8 \\
\hline $\mathrm{CH}_{3} \mathrm{NO}_{2}$ & Methane, nitro- & 571.1 & 572.9 & 572.8 & 300.3 & 214.3 & 570.1 & 570.1 \\
\hline $\mathrm{HCOOCH}_{3}$ & Methyl formate & 748.3 & 749.2 & 745.9 & 501.6 & 447.6 & 746.3 & 746.3 \\
\hline \multicolumn{9}{|l|}{ VALIDATION SET } \\
\hline $\mathrm{C}_{4} \mathrm{H}_{10}$ & Isobutane & 1221.3 & 1229.4 & 1218.3 & 911.4 & 882.9 & 1119.3 & 1221.4 \\
\hline $\mathrm{CH}_{3} \mathrm{NH}_{2}$ & Methyl amine & 541.7 & 542.0 & 540.6 & 366.1 & 342.7 & 477.7 & 542.5 \\
\hline $\mathrm{CH}_{3} \mathrm{CONH}_{2}$ & Acetamide & 822.2 & 824.2 & 822.7 & 548.9 & 498.3 & 755.6 & 822.9 \\
\hline $\mathrm{CH}_{3} \mathrm{CH}_{2} \mathrm{NH}_{2}$ & Ethylamine & 820.0 & 822.8 & 818.7 & 573.1 & 542.5 & 735.8 & 819.9 \\
\hline $\mathrm{CH}_{3} \mathrm{COCH}_{3}$ & Acetone & 927.0 & 931.9 & 926.2 & 662.9 & 617.0 & 862.2 & 926.8 \\
\hline $\mathrm{C}_{2} \mathrm{H}_{4} \mathrm{NH}$ & Aziridine & 675.7 & 680.7 & 678.3 & 454.5 & 410.8 & 613.3 & 676.5 \\
\hline $\mathrm{C}_{2} \mathrm{H}_{5} \mathrm{OCH}_{3}$ & Ethane,methoxy- & 1029.0 & 1033.5 & 1025.6 & 737.9 & 699.6 & 942.9 & 1027.9 \\
\hline $\mathrm{CH}_{3} \mathrm{CH}_{2} \mathrm{OH}$ & Ethanol & 760.7 & 761.0 & 757.1 & 536.3 & 504.7 & 691.8 & 760.6 \\
\hline $\mathrm{CH}_{3} \mathrm{OCH}_{3}$ & Dimethyl ether & 749.0 & 751.4 & 746.2 & 529.3 & 498.7 & 683.3 & 748.4 \\
\hline $\mathrm{CH}_{3} \mathrm{CH}_{2} \mathrm{O}\left({ }^{2} \mathrm{~A}^{\prime \prime}\right)$ & Ethoxy radical & 656.0 & 665.5 & 660.4 & 473.2 & 445.3 & 597.6 & 657.2 \\
\hline
\end{tabular}

a)Experimental atomization energy are from experimental enthalpy of formation. ${ }^{1-3}$ 
Table A2. Zero point energy corrections for different levels of theory

\begin{tabular}{|c|c|c|c|c|c|c|c|}
\hline Molecule & Name & $\begin{array}{l}\text { G3x } \\
\text { (Ha) } \\
\end{array}$ & $\begin{array}{c}\text { B3PW91/ } \\
6-31 \mathrm{G}^{* *}(\mathrm{Ha}) \\
\end{array}$ & $\begin{array}{c}\text { B3PW91/ } \\
\text { cc-pVTZ (Ha) } \\
\end{array}$ & $\begin{array}{c}\mathrm{HF} / \\
6-31 \mathrm{G}^{*}(\mathrm{Ha}) \\
\end{array}$ & $\begin{array}{c}\mathrm{HF} / \\
3-21 \mathrm{G}(\mathrm{Ha}) \\
\end{array}$ & $\begin{array}{c}\text { MP2(full)/ } \\
6-31 G^{*}(\mathrm{Ha}) \\
\end{array}$ \\
\hline \multicolumn{8}{|l|}{ TRAINING SET } \\
\hline $\mathrm{H}_{2}$ & Hydrogen gas & 0.01003 & 0.01015 & 0.01005 & 0.01059 & 0.01062 & 0.01033 \\
\hline $\mathrm{CH}\left({ }^{2} \mathrm{P}\right)$ & Methylidyne & 0.00632 & 0.00638 & 0.00643 & 0.00697 & 0.00656 & 0.00670 \\
\hline $\mathrm{CH}_{2}\left({ }^{3} \mathrm{~B}_{1}\right)$ & Methylene & 0.01705 & 0.01742 & 0.01722 & 0.01843 & 0.01813 & 0.01803 \\
\hline $\mathrm{CH}_{2}\left({ }^{1} \mathrm{~A}_{1}\right)$ & Methylene & 0.01632 & 0.01655 & 0.01656 & 0.01796 & 0.01751 & 0.01727 \\
\hline $\mathrm{CH}_{3}\left({ }^{2} \mathrm{~A}_{2}\right)$ & Methyl radical & 0.02922 & 0.02982 & 0.02966 & 0.03097 & 0.03105 & 0.03055 \\
\hline $\mathrm{CH}_{4}$ & Methane & 0.04411 & 0.04508 & 0.04461 & 0.04780 & 0.04803 & 0.04637 \\
\hline $\mathrm{NH}\left({ }^{3} \mathrm{~S}_{\mathrm{g}}\right)$ & Imidogen & 0.00730 & 0.00746 & 0.00747 & 0.00804 & 0.00721 & 0.00767 \\
\hline $\mathrm{NH}_{2}\left({ }^{2} \mathrm{~B}_{1}\right)$ & Amino radical & 0.01871 & 0.01912 & 0.01906 & 0.02057 & 0.01955 & 0.01960 \\
\hline NH3 & Ammonia & 0.03387 & 0.03465 & 0.03440 & 0.03701 & 0.03603 & 0.03530 \\
\hline $\mathrm{OH}$ & Hydroxyl radical & 0.00829 & 0.00852 & 0.00851 & 0.00911 & 0.00822 & 0.00852 \\
\hline $\mathrm{OH}_{2}$ & Water & 0.02106 & 0.02157 & 0.02145 & 0.02296 & 0.02177 & 0.02148 \\
\hline $\mathrm{HCCH}$ & Acetylene & 0.02645 & 0.02685 & 0.02704 & 0.02945 & 0.03005 & 0.02586 \\
\hline $\mathrm{H}_{2} \mathrm{CCH}_{2}$ & Ethylene & 0.05025 & 0.05119 & 0.05092 & 0.05477 & 0.05509 & 0.05208 \\
\hline $\mathrm{H}_{3} \mathrm{CCH}_{3}$ & Ethane & 0.07345 & 0.07505 & 0.07443 & 0.07978 & 0.08005 & 0.07721 \\
\hline $\mathrm{CN}(2)$ & Cyano radical & 0.00481 & 0.00496 & 0.00493 & 0.00452 & 0.00413 & 0.00654 \\
\hline $\mathrm{HCN}$ & Hydrogen cyanide & 0.01636 & 0.01475 & 0.01463 & 0.01799 & 0.01838 & 0.01599 \\
\hline $\mathrm{CO}$ & Carbon monoxide & 0.00495 & 0.00505 & 0.00506 & 0.00556 & 0.00528 & 0.00484 \\
\hline $\mathrm{HCO}\left({ }^{2} \mathrm{~A}^{\prime}\right)$ & Formyl radical & 0.01280 & 0.01313 & 0.01303 & 0.01438 & 0.01402 & -0.00052 \\
\hline $\mathrm{H}_{2} \mathrm{CO}$ & Formaldehyde & 0.02622 & 0.02679 & 0.02655 & 0.02921 & 0.02898 & -0.00052 \\
\hline $\mathrm{H}_{3} \mathrm{COH}$ & Methyl alcohol & 0.05045 & 0.05161 & 0.05119 & 0.05534 & 0.05449 & 0.05262 \\
\hline $\mathrm{N}_{2}$ & Nitrogen gas & 0.00546 & 0.00563 & 0.00561 & 0.00628 & 0.00595 & 0.00497 \\
\hline $\mathrm{H}_{2} \mathrm{NNH}_{2}$ & Hydrazine & 0.05254 & 0.05370 & 0.05350 & 0.05807 & 0.05627 & 0.05470 \\
\hline $\mathrm{NO}(2)$ & Nitric oxide & 0.00446 & 0.00460 & 0.00456 & 0.00506 & 0.00172 & 0.00887 \\
\hline $\mathrm{O}_{2}\left({ }^{3} \mathrm{~S}_{\mathrm{gg}}\right)$ & Oxygen gas & 0.00374 & 0.00387 & 0.00381 & 0.00455 & 0.00369 & 0.00322 \\
\hline $\mathrm{HOOH}$ & Hydrogen peroxide & 0.02614 & 0.02674 & 0.02676 & 0.02930 & 0.02700 & 0.02628 \\
\hline $\mathrm{CO}_{2}$ & Carbon dioxide & 0.01166 & 0.01171 & 0.01179 & 0.01275 & 0.01186 & 0.01156 \\
\hline $\mathrm{CH}_{2}=\mathrm{C}=\mathrm{CH}_{2}$ & Allene & 0.05460 & 0.05568 & 0.05554 & 0.06010 & 0.06089 & 0.05573 \\
\hline $\mathrm{CH}_{3} \mathrm{CCH}$ & Propyne & 0.05438 & 0.05536 & 0.05494 & 0.05945 & 0.05986 & \multirow{2}{*}{$\begin{array}{l}0.05633 \\
0.05729\end{array}$} \\
\hline $\mathrm{C}_{3} \mathrm{H}_{4}$ & Cyclopropene & 0.05539 & 0.05637 & 0.05601 & 0.06052 & 0.06070 & \\
\hline $\mathrm{CH}_{3} \mathrm{CH}=\mathrm{CH}_{2}$ & Propylene & 0.07840 & 0.07995 & 0.07945 & 0.08545 & 0.08581 & 0.08164 \\
\hline $\mathrm{C}_{3} \mathrm{H}_{6}$ & Cyclopropane & 0.08003 & 0.08173 & 0.08121 & 0.08726 & 0.08730 & 0.08391 \\
\hline $\mathrm{C}_{3} \mathrm{H}_{8}$ & Propane & 0.10168 & 0.10389 & 0.10307 & 0.11058 & 0.11094 & 0.10681 \\
\hline $\mathrm{CH}_{2} \mathrm{CHCHCH}_{2}$ & Butadiene & 0.08387 & 0.08540 & 0.08494 & 0.09154 & 0.09205 & \multirow{2}{*}{$\begin{array}{l}0.08646 \\
0.08539\end{array}$} \\
\hline $\mathrm{C}_{4} \mathrm{H}_{6}$ & 2-butyne & 0.08268 & 0.08433 & 0.08393 & 0.09060 & 0.09149 & \\
\hline $\mathrm{C}_{4} \mathrm{H}_{6}$ & Methylene cyclopropane & 0.08387 & 0.08551 & 0.08500 & 0.09175 & 0.09213 & 0.08734 \\
\hline $\mathrm{C}_{4} \mathrm{H}_{6}$ & Bicyclobutane & 0.08501 & 0.08696 & 0.08635 & 0.09310 & 0.09312 & 0.08870 \\
\hline $\mathrm{C}_{4} \mathrm{H}_{6}$ & Cyclobutene & 0.08513 & 0.08693 & 0.08634 & 0.09323 & 0.09304 & 0.08847 \\
\hline $\mathrm{C}_{4} \mathrm{H}_{8}$ & Cycloctane & 0.10894 & 0.11137 & 0.11059 & 0.11880 & 0.11850 & 0.11416 \\
\hline $\mathrm{C}_{4} \mathrm{H}_{8}$ & Isobutene & 0.10608 & 0.10820 & 0.10742 & 0.11566 & 0.11599 & 0.11068 \\
\hline $\mathrm{C}_{4} \mathrm{H}_{10}$ & Trans butane & 0.12971 & 0.13250 & 0.13149 & 0.14117 & 0.14157 & 0.13616 \\
\hline $\mathrm{C}_{5} \mathrm{H}_{8}$ & Spiropentane & 0.11316 & 0.11556 & 0.11492 & 0.12372 & 0.12353 & 0.11838 \\
\hline $\mathrm{CH}_{3} \mathrm{CN}$ & Methyl cyanide & 0.04462 & 0.04552 & 0.04520 & 0.04890 & 0.04938 & 0.04607 \\
\hline $\mathrm{CH}_{3} \mathrm{ONO}$ & Methyl nitrite & 0.04774 & 0.04893 & 0.04847 & 0.05370 & 0.05979 & 0.04969 \\
\hline $\mathrm{HCOOH}$ & Formic acid & 0.03340 & 0.03419 & 0.03389 & 0.03705 & 0.04037 & 0.03428 \\
\hline $\mathrm{NCCN}$ & Cyanogen & 0.01631 & 0.01657 & 0.01644 & 0.01820 & 0.01932 & 0.01550 \\
\hline
\end{tabular}




\begin{tabular}{|c|c|c|c|c|c|c|c|}
\hline Molecule & Name & $\begin{array}{l}\mathrm{G} 3 \mathrm{x} \\
(\mathrm{Ha}) \\
\end{array}$ & $\begin{array}{c}\text { B3PW91/ } \\
\text { 6-31G** (Ha) }\end{array}$ & $\begin{array}{c}\text { B3PW91/ } \\
\text { cc-pVTZ (Ha) }\end{array}$ & $\begin{array}{c}\mathrm{HF} / \\
6-31 \mathrm{G}^{*}(\mathrm{Ha}) \\
\end{array}$ & $\begin{array}{c}\mathrm{HF} / \\
3-21 \mathrm{G}(\mathrm{Ha}) \\
\end{array}$ & $\begin{array}{c}\text { MP2(full)/ } \\
\text { 6-31G* (Ha) }\end{array}$ \\
\hline$\left(\mathrm{CH}_{3}\right)_{2} \mathrm{NH}$ & Dimethylamine & 0.09087 & 0.09286 & 0.09209 & 0.09945 & 0.09876 & 0.09531 \\
\hline $\mathrm{CH}_{2} \mathrm{CO}$ & Ketene & 0.03123 & 0.03179 & 0.03157 & 0.03421 & 0.03476 & 0.03209 \\
\hline $\mathrm{C}_{2} \mathrm{H}_{4} \mathrm{O}$ & Oxirane & 0.05645 & 0.05772 & 0.05741 & 0.06228 & 0.06140 & 0.05891 \\
\hline $\mathrm{CH}_{3} \mathrm{CHO}$ & Acetaldehyde & 0.05455 & 0.05570 & 0.05529 & 0.05993 & 0.05979 & 0.05700 \\
\hline $\mathrm{HCOCOH}$ & Glyoxal & 0.03641 & 0.03717 & 0.03693 & 0.04085 & 0.04037 & 0.03753 \\
\hline $\mathrm{CH}_{2}=\mathrm{CHCN}$ & Acrylonitrile & 0.05006 & 0.05097 & 0.05072 & 0.05495 & 0.05550 & 0.05108 \\
\hline $\mathrm{CH}_{3} \mathrm{COOH}$ & Acetic acid & 0.06083 & 0.06222 & 0.09810 & 0.06687 & 0.06596 & 0.06317 \\
\hline$\left(\mathrm{CH}_{3}\right)_{2} \mathrm{CHOH}$ & Isopropanol & 0.10610 & 0.10853 & 0.10766 & 0.11599 & 0.11535 & 0.11106 \\
\hline$\left(\mathrm{CH}_{3}\right)_{3} \mathrm{~N}$ & Trimethylamine & 0.11820 & 0.12075 & 0.11969 & 0.12950 & 0.12907 & 0.12409 \\
\hline $\mathrm{C}_{4} \mathrm{H}_{4} \mathrm{O}$ & Furan & 0.06886 & 0.07045 & 0.07010 & 0.07573 & 0.07575 & 0.07054 \\
\hline $\mathrm{C}_{4} \mathrm{H}_{5} \mathrm{~N}$ & Pyrrole & 0.08123 & 0.08302 & 0.08267 & 0.08877 & 0.08957 & 0.08318 \\
\hline $\mathrm{C}_{5} \mathrm{H}_{5} \mathrm{~N}$ & Pyridine & 0.08738 & 0.08913 & 0.08875 & 0.09544 & 0.09631 & 0.08964 \\
\hline $\mathrm{CCH}\left({ }^{2} \mathrm{~A}^{\prime}\right)$ & Ethynyl radical & 0.01301 & 0.01423 & 0.01463 & 0.01501 & 0.01499 & \multirow{2}{*}{$\begin{array}{l}0.01764 \\
0.03889\end{array}$} \\
\hline $\mathrm{C}_{2} \mathrm{H}_{3}\left({ }^{2} \mathrm{~A}^{\prime}\right)$ & Vinyl & 0.03598 & 0.03677 & 0.03647 & 0.03871 & 0.03872 & \\
\hline $\mathrm{CH}_{3} \mathrm{CO}\left({ }^{2} \mathrm{~A}^{\prime}\right)$ & Acetyl radical & 0.04241 & 0.04334 & 0.04306 & 0.04655 & 0.04622 & 0.04448 \\
\hline $\mathrm{H}_{2} \mathrm{COH}\left({ }^{2} \mathrm{~A}\right)$ & Hydroxymethyl radical & 0.03686 & 0.03767 & 0.03724 & 0.04022 & 0.03898 & 0.03846 \\
\hline $\mathrm{C}_{2} \mathrm{H}_{5}\left({ }^{2} \mathrm{~A}^{\prime}\right)$ & Ethyl radical & 0.05827 & 0.05958 & 0.05911 & 0.06335 & 0.06321 & 0.06149 \\
\hline$\left(\mathrm{CH}_{3}\right)_{2} \mathrm{CH}\left({ }^{2} \mathrm{~A}^{\prime}\right)$ & Isopropyl radical & 0.08652 & 0.08848 & 0.08770 & 0.09455 & 0.09445 & 0.09139 \\
\hline$\left(\mathrm{CH}_{3}\right)_{3} \mathrm{C}\left({ }^{2} \mathrm{~A}_{1}\right)$ & t-butyl radical & 0.11443 & 0.11704 & 0.11611 & 0.12522 & 0.12540 & 0.12085 \\
\hline $\mathrm{NO}_{2}(2)$ & Nitrogen dioxide & 0.00871 & 0.00902 & 0.00898 & 0.00985 & 0.00748 & \multirow{2}{*}{$\begin{array}{l}0.01008 \\
0.08603\end{array}$} \\
\hline $\mathrm{C}_{4} \mathrm{H}_{6}$ & Methyl allene & 0.08269 & 0.08430 & 0.08371 & 0.09032 & 0.09069 & \\
\hline $\mathrm{C}_{5} \mathrm{H}_{8}$ & Isoprene & 0.11176 & 0.11385 & 0.11318 & 0.12196 & 0.12193 & 0.11570 \\
\hline $\mathrm{C}_{5} \mathrm{H}_{10}$ & Cyclopentane & 0.13836 & 0.14134 & 0.14048 & 0.15072 & 0.15086 & 0.14467 \\
\hline $\mathrm{C}_{5} \mathrm{H}_{12}$ & n-pentane & 0.15780 & 0.16118 & 0.15999 & 0.17172 & 0.17218 & \multirow{2}{*}{$\begin{array}{l}0.16548 \\
0.16436\end{array}$} \\
\hline $\mathrm{C}_{5} \mathrm{H}_{12}$ & Neo pentane & 0.15715 & 0.16066 & 0.15945 & 0.17065 & 0.17118 & \\
\hline $\mathrm{C}_{6} \mathrm{H}_{8}$ & 1,3 cyclohexadiene & 0.12029 & 0.12273 & 0.12214 & 0.13165 & 0.13205 & 0.12473 \\
\hline $\mathrm{C}_{6} \mathrm{H}_{8}$ & 1,4 cyclohexadiene & 0.12004 & 0.12248 & 0.12198 & 0.13152 & 0.13186 & 0.12464 \\
\hline $\mathrm{C}_{6} \mathrm{H}_{12}$ & Cyclohexane & 0.16722 & 0.17078 & 0.16969 & 0.18243 & 0.18281 & 0.17514 \\
\hline $\mathrm{C}_{6} \mathrm{H}_{14}$ & n-hexane & 0.18574 & 0.18969 & 0.18832 & 0.20224 & 0.20274 & 0.19476 \\
\hline $\mathrm{C}_{6} \mathrm{H}_{14}$ & 3-methyl pentane & 0.18533 & 0.18923 & 0.18786 & 0.20201 & \multirow{2}{*}{$\begin{array}{l}0.20251 \\
0.13797\end{array}$} & 0.19440 \\
\hline $\mathrm{C}_{6} \mathrm{H}_{5} \mathrm{CH}_{3}$ & Toluene & 0.12556 & 0.12826 & 0.12767 & 0.13694 & & \multirow{2}{*}{$\begin{array}{l}0.12921 \\
0.22403\end{array}$} \\
\hline $\mathrm{C}_{7} \mathrm{H}_{16}$ & n-heptane & 0.21382 & 0.21837 & 0.21681 & 0.23274 & 0.23329 & \\
\hline $\mathrm{C}_{8} \mathrm{H}_{8}$ & Cyclooctatetraene & 0.13036 & 0.13292 & 0.13248 & 0.14338 & \multirow{2}{*}{$\begin{array}{l}0.14364 \\
0.26382\end{array}$} & \multirow{2}{*}{$\begin{array}{l}0.13390 \\
0.25328\end{array}$} \\
\hline $\mathrm{C}_{8} \mathrm{H}_{18}$ & n-octane & 0.24172 & 0.24684 & 0.24512 & 0.26323 & & \\
\hline $\mathrm{C}_{10} \mathrm{H}_{8}$ & Naphthalene & 0.14480 & 0.14799 & 0.14747 & 0.15816 & \multirow{2}{*}{$\begin{array}{l}0.15963 \\
0.15579\end{array}$} & \multirow{2}{*}{0.14709} \\
\hline $\mathrm{C}_{10} \mathrm{H}_{8}$ & Azulene & 0.14368 & 0.14666 & 0.14597 & 0.15465 & & \\
\hline $\mathrm{CH}_{3} \mathrm{COOCH}_{3}$ & Acetic acid methyl ester & 0.08811 & 0.09006 & 0.08942 & 0.09706 & 0.09630 & 0.09226 \\
\hline$\left(\mathrm{CH}_{3}\right)_{3} \mathrm{COH}$ & t-butanol & 0.13310 & 0.13621 & 0.13505 & 0.14558 & 0.14499 & 0.13953 \\
\hline $\mathrm{C}_{6} \mathrm{H}_{5} \mathrm{NH}_{2}$ & Aniline & 0.11515 & 0.11759 & 0.11715 & 0.12561 & 0.12588 & \multirow{2}{*}{$\begin{array}{l}0.11776 \\
0.10483\end{array}$} \\
\hline $\mathrm{C}_{6} \mathrm{H}_{5} \mathrm{OH}$ & Phenol & 0.10297 & 0.10516 & 0.10480 & 0.11230 & 0.11284 & \\
\hline $\mathrm{C}_{4} \mathrm{H}_{6} \mathrm{O}$ & Divinyl ether & 0.08783 & 0.08943 & 0.08896 & 0.09608 & 0.09708 & 0.09028 \\
\hline $\mathrm{C}_{4} \mathrm{H}_{8} \mathrm{O}$ & Tetrahydrofuran & 0.11472 & 0.11728 & 0.11659 & 0.12592 & 0.12546 & 0.12001 \\
\hline $\mathrm{C}_{5} \mathrm{H}_{8} \mathrm{O}$ & Cyclopentanone & 0.11921 & 0.12182 & 0.12109 & 0.13052 & \multirow{2}{*}{$\begin{array}{l}0.13037 \\
0.09305\end{array}$} & \multirow{2}{*}{$\begin{array}{l}0.12448 \\
0.08515\end{array}$} \\
\hline $\mathrm{C}_{6} \mathrm{H}_{4} \mathrm{O}_{2}$ & Benzoquinone & 0.08368 & 0.08540 & 0.08517 & 0.09243 & & \\
\hline $\mathrm{C}_{4} \mathrm{H}_{4} \mathrm{~N}_{2}$ & Pyrimidine & 0.07581 & 0.07736 & 0.08517 & 0.08327 & 0.08343 & 0.07798 \\
\hline $\mathrm{N}=\mathrm{C}-\mathrm{CH}_{2}-\mathrm{CH}_{2}-\mathrm{C}=\mathrm{N}$ & Butanedinitrile & 0.07231 & 0.07377 & 0.07337 & 0.07960 & 0.08029 & 0.07419 \\
\hline $\mathrm{C}_{4} \mathrm{H}_{4} \mathrm{~N}_{2}$ & 1,4-dipyridine & 0.07563 & 0.07714 & 0.07681 & 0.08295 & \multirow{2}{*}{$\begin{array}{l}0.08326 \\
0.07161\end{array}$} & \multirow{2}{*}{$\begin{array}{l}0.07776 \\
0.06602\end{array}$} \\
\hline $\mathrm{CH}_{3}-\mathrm{C}(=\mathrm{O})-\mathrm{CCH}$ & & 0.06418 & 0.06541 & 0.06512 & 0.07086 & & \\
\hline $\mathrm{CH}_{3}-\mathrm{CH}=\mathrm{CH}-\mathrm{CHO}$ & Crotonaldehyde & 0.08812 & 0.08987 & 0.08931 & 0.09664 & 0.09668 & 0.09149 \\
\hline
\end{tabular}




\begin{tabular}{|c|c|c|c|c|c|c|c|}
\hline Molecule & Name & $\begin{array}{l}\mathrm{G} 3 \mathrm{x} \\
(\mathrm{Ha}) \\
\end{array}$ & $\begin{array}{c}\text { B3PW91/ } \\
6-31 \mathrm{G}^{* *}(\mathrm{Ha}) \\
\end{array}$ & $\begin{array}{c}\text { B3PW91/ } \\
\text { cc-pVTZ (Ha) }\end{array}$ & $\begin{array}{c}\mathrm{HF} / \\
6-31 \mathrm{G}^{*}(\mathrm{Ha}) \\
\end{array}$ & $\begin{array}{c}\mathrm{HF} / \\
3-21 \mathrm{G}(\mathrm{Ha}) \\
\end{array}$ & $\begin{array}{c}\text { MP2(full)/ } \\
\text { 6-31G* (Ha) }\end{array}$ \\
\hline $\mathrm{CH}_{3}-\mathrm{C}(=\mathrm{O})-\mathrm{O}-\mathrm{C}(=\mathrm{O})-\mathrm{CH}_{3}$ & Acetic anhydride & 0.09663 & 0.09875 & 0.09810 & 0.10644 & 0.10590 & 0.10072 \\
\hline$\left(\mathrm{CH}_{3}\right)_{2} \mathrm{CH}-\mathrm{CN}$ & Propanenitrile, 2-methyl & 0.10082 & 0.10298 & 0.10227 & 0.11017 & 0.11066 & 0.10503 \\
\hline $\mathrm{CH}_{3}-\mathrm{CO}-\mathrm{CH}_{2}-\mathrm{CH}_{3}$ & Methyl ethyl ketone & 0.11037 & 0.11270 & 0.11187 & -0.00105 & 0.12087 & 0.11560 \\
\hline$\left(\mathrm{CH}_{3}\right)_{2} \mathrm{CH}-\mathrm{CHO}$ & Propanal,2-methyl & 0.11064 & 0.11302 & 0.11218 & 0.12110 & 0.12117 & 0.11571 \\
\hline $\mathrm{C}_{4} \mathrm{H}_{8} \mathrm{O}_{2}$ & 1,4-Dioxane & 0.12017 & 0.12289 & 0.12200 & 0.13276 & 0.13154 & 0.12577 \\
\hline $\mathrm{C}_{4} \mathrm{H}_{8} \mathrm{NH}$ & Tetrahydropyrrole & 0.12751 & 0.13027 & 0.12953 & 0.13952 & 0.13842 & 0.13315 \\
\hline $\mathrm{CH}_{3}-\mathrm{CH}_{2}-\mathrm{CH}\left(\mathrm{CH}_{3}\right)-\mathrm{NO}_{2}$ & Nitro-s-butane & 0.13295 & 0.13601 & 0.13508 & 0.14614 & 0.14393 & 0.13936 \\
\hline $\mathrm{CH}_{3}-\mathrm{CH}_{2}-\mathrm{O}-\mathrm{CH}_{2}-\mathrm{CH}_{3}$ & Diethyl ether & 0.13417 & 0.13716 & 0.13607 & 0.14690 & 0.14666 & 0.14081 \\
\hline $\mathrm{CH}_{3}-\mathrm{CH}\left(\mathrm{OCH}_{3}\right)_{2}$ & dimethyl acetal & 0.13875 & 0.14185 & 0.14070 & \multirow{2}{*}{$\begin{array}{l}0.15192 \\
0.15943\end{array}$} & \multirow{2}{*}{$\begin{array}{l}0.15083 \\
0.15877\end{array}$} & \multirow{2}{*}{$\begin{array}{l}0.14484 \\
0.15296\end{array}$} \\
\hline $\begin{array}{l}\left(\mathrm{CH}_{3}\right)_{3} \mathrm{C}-\mathrm{NH}_{2} \\
\text { cyc-CH=CH-N }\left(\mathrm{CH}_{3}\right)-\end{array}$ & t-butylamine & 0.14567 & 0.14897 & 0.14774 & & & \\
\hline $\mathrm{CH}=\mathrm{CH}-$ & N-methyl pyrrole & 0.10840 & 0.11082 & 0.11022 & 0.11864 & 0.11907 & 0.11191 \\
\hline $\mathrm{C}_{5} \mathrm{H}_{10} \mathrm{O}$ & Tetrahydropyran & 0.14384 & 0.14696 & 0.14596 & 0.15764 & 0.15725 & 0.15053 \\
\hline $\mathrm{CH}_{3}-\mathrm{CH}_{2}-\mathrm{CO}-\mathrm{CH}_{2}-\mathrm{CH}_{3}$ & Diethyl ketone & 0.13850 & 0.14149 & 0.14055 & 0.15156 & \multirow{2}{*}{$\begin{array}{l}0.15177 \\
0.15686\end{array}$} & 0.14519 \\
\hline $\mathrm{C}_{5} \mathrm{H}_{10} \mathrm{O}_{2}$ & Isopropyl acetate & 0.14342 & 0.14661 & 0.14548 & 0.15720 & & 0.15028 \\
\hline cyc- $\mathrm{C}_{5} \mathrm{H}_{10} \mathrm{NH}$ & Piperidine & 0.15619 & 0.15958 & 0.15850 & 0.17110 & 0.17052 & 0.16352 \\
\hline$\left(\mathrm{CH}_{3}\right)_{2} \mathrm{CH}-\mathrm{O}-\mathrm{CH}\left(\mathrm{CH}_{3}\right)_{2}$ & Di-isopropyl ether & 0.18894 & 0.19316 & 0.19164 & 0.20659 & 0.20646 & \multirow{2}{*}{0.09271} \\
\hline $\mathrm{C}_{6} \mathrm{H}_{5}\left({ }^{2} \mathrm{~A}_{1}\right)$ & Phenyl radical & 0.08589 & 0.08775 & 0.08740 & 0.09156 & 0.09219 & \\
\hline$\left(\mathrm{CH}_{3}\right)_{3} \mathrm{C}-\mathrm{O}-\mathrm{CH}_{3}$ & t-butyl methyl ether & 0.16084 & 0.16433 & 0.16304 & 0.17607 & 0.17584 & 0.16879 \\
\hline $\mathrm{C}_{6} \mathrm{H}_{6}$ & Benzene & 0.09869 & 0.10083 & 0.10050 & 0.10767 & 0.10881 & 0.10087 \\
\hline $\mathrm{CH}_{3} \mathrm{NO}_{2}$ & Methane, nitro- & 0.04907 & 0.05026 & 0.04984 & 0.05458 & 0.05193 & 0.05139 \\
\hline $\mathrm{HCOOCH}_{3}$ & Methyl formate & 0.06104 & 0.06242 & 0.06191 & 0.06757 & 0.06668 & 0.06317 \\
\hline \multicolumn{8}{|l|}{ VALIDATION SET } \\
\hline $\mathrm{C}_{4} \mathrm{H}_{10}$ & Isobutane & 0.12928 & 0.13207 & 0.13104 & 0.14077 & 0.14123 & 0.13574 \\
\hline $\mathrm{CH}_{3} \mathrm{NH}_{2}$ & Methyl amine & 0.06307 & 0.06446 & 0.06396 & 0.06891 & 0.06802 & 0.06599 \\
\hline $\mathrm{CH}_{3} \mathrm{CONH}_{2}$ & Acetamide & 0.07209 & 0.07376 & 0.07325 & 0.07898 & 0.07953 & 0.07516 \\
\hline $\mathrm{CH}_{3} \mathrm{CH}_{2} \mathrm{NH}_{2}$ & Ethylamine & 0.09124 & 0.09326 & 0.09252 & 0.09958 & 0.09877 & 0.09555 \\
\hline $\mathrm{CH}_{3} \mathrm{COCH}_{3}$ & Acetone & 0.08214 & 0.08382 & 0.08316 & 0.08992 & 0.08992 & 0.08599 \\
\hline $\mathrm{C}_{2} \mathrm{H}_{4} \mathrm{NH}$ & Aziridine & 0.06913 & 0.07063 & 0.07026 & 0.07595 & 0.07485 & 0.07216 \\
\hline $\mathrm{C}_{2} \mathrm{H}_{5} \mathrm{OCH}_{3}$ & Ethane,methoxy- & 0.10630 & 0.10865 & 0.10776 & 0.11655 & 0.11614 & 0.11156 \\
\hline $\mathrm{CH}_{3} \mathrm{CH}_{2} \mathrm{OH}$ & Ethanol & 0.07859 & 0.08041 & 0.07981 & 0.08602 & 0.08533 & 0.08217 \\
\hline $\mathrm{CH}_{3} \mathrm{OCH}_{3}$ & Dimethyl ether & 0.07837 & 0.08008 & 0.07941 & 0.08615 & 0.08557 & 0.08228 \\
\hline $\mathrm{CH}_{3} \mathrm{CH}_{2} \mathrm{O}\left({ }^{2} \mathrm{~A}^{\prime \prime}\right)$ & Ethoxy radical & 0.06482 & 0.06438 & 0.06398 & 0.07008 & 0.07003 & 0.06858 \\
\hline
\end{tabular}

\section{References}

(1) Curtiss, L. A.; Raghavachari, K.; Redfern, P. C.; Rassolov, V.; Pople, J. A. J. Chem. Phys. 1998, 109, 7764.

(2) Curtiss, L. A.; Raghavachari, K.; Redfern, P. C.; Pople, J. A. J. Chem. Phys. 2000, 112,7374 .

(3) Curtiss, L. A.; Redfern, P. C.; Raghavachari, K. The Journal of Chemical Physics 2005, 123, 124107. 